\title{
Joint Density for Eigenvalues of Two Correlated Complex Wishart Matrices: Characterization of MIMO Systems
}

\author{
Ping-Heng Kuo, Student Member, IEEE, Peter J. Smith, Senior Member, IEEE, \\ and Lee M. Garth, Senior Member, IEEE
}

\begin{abstract}
In this letter, the joint probability density function (PDF) for the eigenvalues of a complex Wishart matrix and a perturbed version of it are derived. The latter version can be used to model channel estimation errors and variations over time or frequency. As an example, the joint PDF is used to calculate the transition probabilities between modulation states in an adaptive MIMO system. This leads to a Markov model for the system. We then use the model to investigate the modulation state entering rates (MSER), the average stay duration (ASD), and the effects of feedback delay on the accuracy of modulation state selection in mobile radio systems. Other applications of this PDF are also discussed.
\end{abstract}

Index Terms-Complex Wishart matrix, eigenvalues, random matrix perturbation, MIMO communication systems, adaptive modulation, Markov models.

\section{INTRODUCTION}

$\mathbf{T}$ HE theory of random matrices has been widely applied in many different areas in science and engineering. In wireless communication, a key application is in the area of multiple-input multiple-output (MIMO) systems. In particular, the eigenvalues of a Wishart matrix (channel correlation matrix) play a very important role in this field, since these eigenvalues are the power gains of the virtual parallel links (eigenmodes) intrinsic to the baseline MIMO channel [1] with i.i.d. Rayleigh fading.

In such MIMO systems, with $T$ antennas at the transmitter and $R$ antennas at the receiver, the channel matrix is often perturbed by the addition of some other complex Gaussian matrix, mathematically expressed as

$$
\widehat{\boldsymbol{H}}=\alpha \boldsymbol{H}+\beta \boldsymbol{A}
$$

where $\widehat{\boldsymbol{H}}, \boldsymbol{H}$ and $\boldsymbol{A}$ are all matrices of the same dimensions $(R \times T)$ with i.i.d. complex zero-mean Gaussian entries. We postulate that the entries of both $\boldsymbol{H}$ and $\boldsymbol{A}$ have unit variances so that the entries are $\operatorname{eN}(0,1)$. The real constants $\alpha$ and $\beta$ determine the variance of $\widehat{\boldsymbol{H}} .^{1}$ If we denote $m=\min (R, T)$ and $n=\max (R, T)$, then there are $m$ non-zero eigenvalues in each of the Wishart matrices $\boldsymbol{H} \boldsymbol{H}^{\dagger}$ and $\widehat{\boldsymbol{H}} \widehat{\boldsymbol{H}}^{\dagger}$. The main

Manuscript received May 31, 2006; revised October 25, 2006; accepted December 2, 2006. The associate editor coordinating the review of this letter and approving it for publication was M. Shafi.

The authors are with the Dept. Electrical and Computer Engineering, University of Canterbury, Christchurch, New Zealand (email: phk15@student.canterbury.ac.nz, \{peter.smith, lee.garth\}@ canterbury.ac.nz). Digital Object Identifier 10.1109/TWC.2007.060309.

${ }^{1}$ Here we restrict $\alpha$ and $\beta$ to be real valued to keep the notation simple. However, to accommodate complex correlation coefficients that arise, for example, in OFDM, we can allow $\alpha$ and $\beta$ to be complex valued, replacing $\rho$ in (3) by $|\rho|$ without any effect on the resulting analysis (as shown in [2]). interest of this letter lies in the joint density function of these $2 m$ non-zero eigenvalues.

Here we enumerate some cases where the perturbation in (1) is encountered in the context of MIMO research:

- Additive channel estimation error: A very common model for channel estimation error is expressed as the additive noise:

$$
\widehat{\boldsymbol{H}}=\boldsymbol{H}+\sqrt{\delta^{2}} \boldsymbol{A}
$$

where $\delta^{2}$ is the variance of the additive error matrix. The exact value of $\delta^{2}$ depends on the estimation method used.

- Imperfectly estimated and outdated CSI: In a realistic system model with channel state information (CSI), imperfect CSI is fed back to the transmitter. Hence, the CSI is imperfectly estimated as (2) at the receiver, and is also outdated when it is used at the transmitter due to feedback delay. As shown in [3] and [4], the estimated/outdated channel (estimated at $t-\tau$ ) in such a scenario can be written as

$$
\widehat{\boldsymbol{H}}_{t-\tau}=\rho \boldsymbol{H}_{t}+\sqrt{\delta_{e}^{2}} \boldsymbol{A}
$$

where $\rho$ is the correlation coefficient between two channel realizations, time $\tau$ apart. Also, $\delta_{e}^{2} \approx 1-|\rho|^{2}+|\rho|^{2} \delta^{2}$, where $\delta^{2}$ is the measure of the estimation error as in (2). This model has been frequently applied in the literature (for example, in [4]).

- Single-tap channel model: The channel time-variation is often modeled by using a "single-tap" filter (for example, in [5]), which is expressed as

$$
\boldsymbol{H}_{t}=\rho \boldsymbol{H}_{t-\tau}+\sqrt{1-\rho^{2}} \boldsymbol{A}
$$

In addition, (4) can be used to model the channel variation in the frequency domain, which is applicable to research on OFDM systems. In this case, $\rho$ would be the modulus value for the complex correlation coefficient between OFDM sub-carriers. Also, some channel estimation error models are realized using (4), where the power is scaled to maintain the variance, in contrast to the models with additive errors described in (2) and (3).

To the best of our knowledge, very little previous work has addressed the joint statistics of the eigenvalues of $\boldsymbol{H} \boldsymbol{H}^{\dagger}$ and $\widehat{\boldsymbol{H}} \widehat{\boldsymbol{H}}^{\dagger}$. In [5], the joint density of the eigenvalues of realizations at two successive time points, $t$ and $t+\tau$, has been derived. This scenario is the same as in (4). In this letter, we derive a more general joint PDF, which caters for any cases that can be expressed in the form of (1). Hence, the result is 
more versatile, but with a much simpler derivation than that found in [5].

In this paper we use the joint statistics to study the temporal behavior of an adaptive MIMO system. As a numerical example, we consider the service process defined in [6] for MIMO systems that merge singular value decomposition (SVD) and adaptive modulation. This service process is modeled as a finite state Markov chain (FSMC) over time. Specifically, we compute the transition probability between states using the PDF. Each of the states corresponds to a set of modulation formats that are used among the multiple eigenmodes. In [6], the FSMC for the service process (or modulation selection) of a MIMO system has been employed in cross-layer design to evaluate the maximum packet arrival rate (effective capacity) at the data-link layer while the prescribed QoS is satisfied. Here, we apply the FSMC model to examine the temporal characteristics of MIMO-SVD systems, including the modulation state entering rates (MSER), the average stay duration (ASD), and the probability of modulation state selection error (MSSE) due to the feedback delay.

The results show that for any initial state, the probability that the channel transits to any other state within a short time period is relatively high. In some states this probability is extremely high. Hence, the impact of feedback delay on the adaptation error performance of the SVD-based MIMO system is particularly significant. Note that a practical Markov channel-based feedback scheme for adaptive modulation has been proposed in [7].

\section{Derivation of the Joint Density}

Denoting the eigenvalues of $\boldsymbol{H} \boldsymbol{H}^{\dagger}$ and $\widehat{\boldsymbol{H}} \widehat{\boldsymbol{H}}^{\dagger}$ by $\lambda_{1}, \ldots, \lambda_{m}$ and $\widehat{\lambda}_{1}, \ldots, \widehat{\lambda}_{m}$ respectively, using Bayes' Rule, the required joint density is:

$$
\begin{aligned}
f\left(\lambda_{1}, \ldots, \lambda_{m}, \widehat{\lambda}_{1}, \ldots, \widehat{\lambda}_{m}\right)= & f\left(\widehat{\lambda}_{1}, \ldots, \widehat{\lambda}_{m} \mid \lambda_{1}, \ldots, \lambda_{m}\right) \\
& \times f\left(\lambda_{1}, \ldots, \lambda_{m}\right) .
\end{aligned}
$$

Since the entries of $\boldsymbol{H}$ are i.i.d. $\mathcal{C N}(0,1), \boldsymbol{H} \boldsymbol{H}^{\dagger}$ is Wishart and the joint density of the ordered eigenvalues is given as [8]:

$$
\begin{aligned}
f\left(\lambda_{1}, \ldots, \lambda_{m}\right)= & \prod_{i=1}^{m}[(n-i) !(m-i) !]^{-1} \exp \left(-\sum_{i=1}^{m} \lambda_{i}\right) \\
& \times \prod_{i=1}^{m} \lambda_{i}^{n-m} \prod_{i<j}\left(\lambda_{i}-\lambda_{j}\right)^{2} .
\end{aligned}
$$

Rewriting (1) as:

$$
\frac{\widehat{\boldsymbol{H}}}{\alpha}=\boldsymbol{H}+\frac{\beta}{\alpha} \boldsymbol{A}
$$

we see that (7) is the sum of two scaled random matrices. This has a similar form to a complex Brownian matrix diffusion process, the structure of which can be expressed as

$$
\boldsymbol{B}_{t+\tau}=\boldsymbol{B}_{t}+\mathcal{C N}(\mathbf{0}, 2 \tau \boldsymbol{I})=\boldsymbol{B}_{t}+\sqrt{2 \tau} \mathcal{C N}(\mathbf{0}, \boldsymbol{I})
$$

with $\tau \geq 0$, where $\boldsymbol{B}_{t}$ represents the $R \times T$ complex Brownian matrix at time point $t$, and $\mathcal{C N}\left(\mathbf{0}, \sigma^{2} \boldsymbol{I}\right)$ denotes an i.i.d. complex Gaussian matrix with entries which have zero mean and magnitude variance $\sigma^{2}$. Note that $\boldsymbol{B}_{t}$ and $\operatorname{e\mathcal {N}}\left(\mathbf{0}, \sigma^{2} \boldsymbol{I}\right)$ are independent. The underlying eigenvalue process of $\boldsymbol{B}_{t}$ has been studied in [9]. Most importantly, it was shown in [9] that the $m$ eigenvalues of $\boldsymbol{B}_{t} \boldsymbol{B}_{t}^{\dagger}$ evolve as $m$ independent Squared Bessel Processes (BESQ) conditioned never to collide. For this system, define one eigenvalue at time $t$ as $w$ and a second, possibly different eigenvalue, at time $t+\tau$ as $\widehat{w}$. The conditional density of $\widehat{w}$ given $w$ is denoted the transition density and is given by [9]:

$$
f(\widehat{w} \mid w)=\frac{1}{2 \tau}\left(\frac{\widehat{w}}{w}\right)^{\frac{v}{2}} \exp \left[\frac{-w-\widehat{w}}{2 \tau}\right] I_{v}\left(\frac{\sqrt{w \widehat{w}}}{\tau}\right)
$$

where $v=n-m$ and $I_{v}$ is the $v^{t h}$-order modified Bessel function.

Since the multiple eigenvalues processes are conditioned never to collide, the ordering $w_{1}>w_{2}>\ldots>w_{m}$ is preserved, and the corresponding joint transition density of the eigenvalues is given as [10]:

$$
f\left(\widehat{w}_{1}, \ldots, \widehat{w}_{m} \mid w_{1}, \ldots, w_{m}\right)=\frac{\prod_{i<j}\left(\widehat{w}_{i}-\widehat{w}_{j}\right)}{\prod_{i<j}\left(w_{i}-w_{j}\right)} \times G(\boldsymbol{w})
$$

where the operator $G(\cdot)$ is the determinant defined as

$$
G(\boldsymbol{x})=\operatorname{det}\left[\begin{array}{cccc}
f\left(\widehat{x}_{1} \mid x_{1}\right) & f\left(\widehat{x}_{2} \mid x_{1}\right) & \cdots & f\left(\widehat{x}_{m} \mid x_{1}\right) \\
f\left(\widehat{x}_{1} \mid x_{2}\right) & f\left(\widehat{x}_{2} \mid x_{2}\right) & & \vdots \\
\vdots & & \ddots & \vdots \\
f\left(\widehat{x}_{1} \mid x_{m}\right) & \cdots & \cdots & f\left(\widehat{x}_{m} \mid x_{m}\right)
\end{array}\right]
$$

for multiple ordered processes $x_{1}, x_{2}, \ldots, x_{m}$.

In order to apply the results for the Brownian model, we replace $w, \widehat{w}$ and $2 \tau$ in (9) with $\lambda, \widehat{\lambda}$ and $(\beta / \alpha)^{2}$ respectively. Then, we multiply (10) with (6) to obtain the joint PDF of the eigenvalues of $\boldsymbol{H} \boldsymbol{H}^{\dagger}$ and $\left(\widehat{\boldsymbol{H}} \widehat{\boldsymbol{H}}^{\dagger}\right) / \alpha^{2}$. Since the eigenvalues of $\widehat{\boldsymbol{H}} \widehat{\boldsymbol{H}}^{\dagger}$ have been scaled by $\alpha^{2}$, we make a simple transformation to obtain the desired results. To recapitulate, we have obtained

$$
f(\widehat{\lambda} \mid \lambda)=\frac{\alpha^{2}}{\beta^{2}}\left(\frac{\widehat{\lambda}}{\alpha^{2} \lambda}\right)^{\frac{v}{2}} \exp \left[\frac{-\alpha^{2} \lambda-\widehat{\lambda}}{\beta^{2}}\right] I_{v}\left(2 \frac{\alpha}{\beta^{2}} \sqrt{\lambda \widehat{\lambda}}\right)
$$

and

$$
\begin{aligned}
f\left(\lambda_{1}, \ldots, \lambda_{m}, \widehat{\lambda}_{1}, \ldots, \widehat{\lambda}_{m}\right) \\
=\frac{\prod_{i<j}\left[\frac{1}{\alpha^{2}}\left(\widehat{\lambda}_{i}-\widehat{\lambda}_{j}\right)\right] \prod_{i<j}\left(\lambda_{i}-\lambda_{j}\right) \prod_{i=1}^{m} \lambda_{i}^{v}}{\alpha^{2 m} \prod_{i=1}^{m}[(n-i) !(m-i) !]} \\
\quad \times \exp \left(-\sum_{i=1}^{m} \lambda_{i}\right) \times G(\boldsymbol{\lambda})
\end{aligned}
$$

where $G(\boldsymbol{\lambda})$ can be calculated based on (11) and (12).

\section{NumERICAL EXAMPLE: A FSMC FOR AN ADAPTIVE Modulation Service Process}

For SVD-based MIMO systems, adaptive modulation over the eigenmodes can be applied to further improve the MIMO system throughput [1]. The service process for an adaptive modulation system is simply the modulation selection over time [6]. Here, we construct a FSMC for the service process by using the joint PDF to compute the transition probabilities 
TABLE I

Possible Modulation Scheme Pairs for Adaptive MiMo Systems WITH TWO EIGENMODES

\begin{tabular}{lll} 
State & $\lambda_{1}$ & $\lambda_{2}$ \\
\hline \hline 1 & Outage & Outage \\
2 & BPSK & Outage \\
3 & BPSK & BPSK \\
4 & QPSK & Outage \\
5 & QPSK & BPSK \\
6 & QPSK & QPSK \\
7 & 8-PSK & Outage \\
8 & 8-PSK & BPSK \\
9 & 8-PSK & QPSK \\
10 & 8-PSK & 8-PSK \\
11 & 16-QAM & Outage \\
12 & 16-QAM & BPSK \\
13 & 16-QAM & QPSK \\
14 & 16-QAM & 8-PSK \\
15 & 16-QAM & 16-QAM \\
\hline
\end{tabular}

between states. Using (4), we set $\alpha=\rho$ and $\beta=\sqrt{1-\rho^{2}}$ for the time-varying channel model. Assuming the time variation is governed by Jakes' model, we have $\rho=J_{0}\left(2 \pi f_{D} \tau\right)$, where $J_{0}(\cdot)$ represents the zeroth-order Bessel function, $f_{D}$ is the Doppler frequency, and $\tau$ is the time displacement. Additionally, $\lambda_{i}$ and $\widehat{\lambda}_{i}$ are the eigenmode gains at time $t$ and $t+\tau$ respectively. We consider a MIMO channel with two antennas at the transmitter and four antennas at the receiver in an i.i.d. Rayleigh flat fading environment $(m=2$ and $n=4)$. The joint PDF (13) for this particular case, after a little algebra, can be written as

$$
\begin{aligned}
f\left(\widehat{\lambda}_{1}, \widehat{\lambda}_{2}, \lambda_{1}, \lambda_{2}\right)= & \frac{\widehat{\lambda}_{1} \widehat{\lambda}_{2} \lambda_{1} \lambda_{2}\left(\widehat{\lambda}_{1}-\widehat{\lambda}_{2}\right)\left(\lambda_{1}-\lambda_{2}\right)}{12\left(\rho^{6}+\rho^{10}-2 \rho^{8}\right)} \\
& \times \exp \left(\frac{-\lambda_{1}-\lambda_{2}-\widehat{\lambda}_{1}-\widehat{\lambda}_{2}}{1-\rho^{2}}\right) \\
& \times\left[I_{2}\left(\frac{2 \rho}{1-\rho^{2}} \sqrt{\lambda_{1} \widehat{\lambda}_{1}}\right) I_{2}\left(\frac{2 \rho}{1-\rho^{2}} \sqrt{\lambda_{2} \widehat{\lambda}_{2}}\right)\right. \\
& \left.-I_{2}\left(\frac{2 \rho}{1-\rho^{2}} \sqrt{\lambda_{1} \hat{\lambda}_{2}}\right) I_{2}\left(\frac{2 \rho}{1-\rho^{2}} \sqrt{\lambda_{2} \widehat{\lambda}_{1}}\right)\right] .
\end{aligned}
$$

Assuming five modulation options: BPSK, QPSK, 8-PSK, 16-QAM or outage (no transmission), there are 15 possible modulation pairs over the two eigenmodes, as tabulated in Table I.

We use particular SNR levels as modulation switching thresholds. These thresholds are the minimum SNR levels required for each modulation scheme to achieve a target BER of $10^{-3}$. Assuming that the transmission power is evenly distributed and the SNR on both eigenmodes is $9 \mathrm{~dB}$ (normalized noise level), the corresponding eigenmode gains for these modulation types can be calculated by approximation methods in [11] (for PSK) and [12] (for square QAM), as summarized in Table II.

To compute the probability, $P_{i j}$, that state $i$ transits to $j$ after time $\tau$, serial integration of the joint PDF is required.
TABLE II

CorResponding EIGENMODE GAIN REGIONS FOR DiFFERENT Modulation Methods

\begin{tabular}{ll} 
Modulation & Corresponding Eigenmode Gain Regions \\
\hline \hline Outage & $0 \leq \lambda<0.3310$ \\
BPSK & $0.3310 \leq \lambda<1.2702$ \\
QPSK & $1.2702 \leq \lambda<4.8738$ \\
8-PSK & $4.8738 \leq \lambda<6.6229$ \\
16-QAM & $6.6229 \leq \lambda$ \\
\hline
\end{tabular}

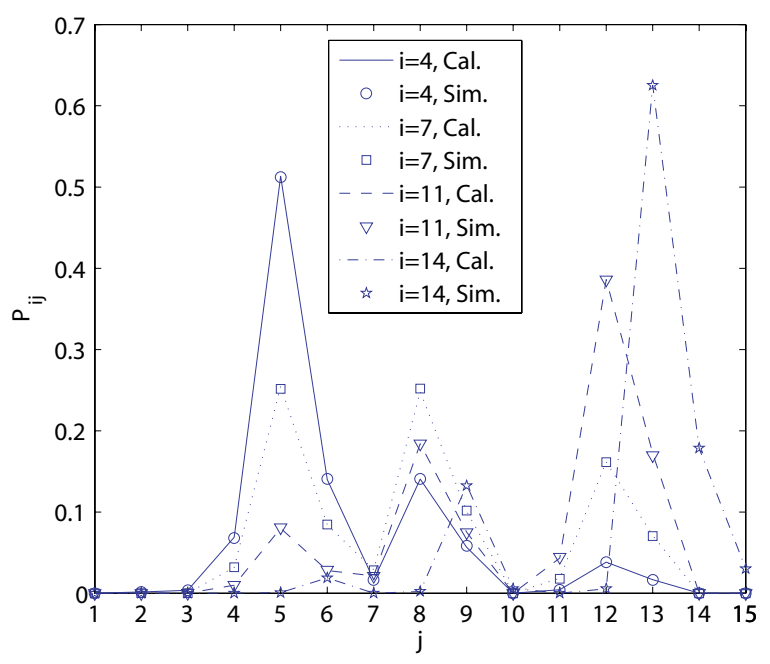

Fig. 1. Simulated vs. calculated transition probabilities from state $i$ to state $j$, with $i=4,7,11$, and 14 . Mobility level $f_{D} \tau=0.1325$.

For example, if $a \leq \lambda_{1}<b, c \leq \lambda_{2}<d$ holds for state $i$, and $e \leq \widehat{\lambda}_{1}<f, g \leq \widehat{\lambda}_{2}<h$ holds for state $j$, then

$$
P_{i j}=\frac{P(i, j)}{P(i)}=\frac{\int_{g}^{h} \int_{c}^{d} \int_{e}^{f} \int_{a}^{b} f\left(\widehat{\lambda}_{1}, \widehat{\lambda}_{2}, \lambda_{1}, \lambda_{2}\right) d \lambda_{1} d \widehat{\lambda}_{1} d \lambda_{2} d \widehat{\lambda}_{2}}{\int_{c}^{d} \int_{a}^{b} f\left(\lambda_{1}, \lambda_{2}\right) d \lambda_{1} d \lambda_{2}} .
$$

Note that $P(i, j)$ and $P(i)$ are the steady state probabilities. The boundary values of the integrals $a, b, \ldots, h$, may depend on $\lambda_{i}$ or $\hat{\lambda}_{i}$. For example, $P_{11}$ involves integrating over the regions $0<\lambda_{2}<\lambda_{1}<0.3310$ and $0<\widehat{\lambda}_{2}<\widehat{\lambda}_{1}<0.3310$. In all cases, calculation of $a, b, \ldots, h$ is straightforward and is omitted here. Hence, by numerical integration of (15) we can calculate the probabilities for all transitions. Selected results are shown in Fig. 1. The simulations were carried out using $f_{D} \tau=0.1325$ and $2 \times 10^{6}$ samples in total. Clearly, the calculations agree well with the simulations. For larger systems, such an approach may become prohibitive due to the need for a large number of integrations. However, for applications where only a few eigenvalues are of interest, the numerical calculations are quite rapid and stable to compute. The transition probabilities in (15) can be used to assess the temporal behavior of the system, as shown in the following sections.

\section{A. MSER and ASD}

In [13], the simultaneous LCR for multiple eigenmodes was approximated by crudely assuming the eigenmodes are 


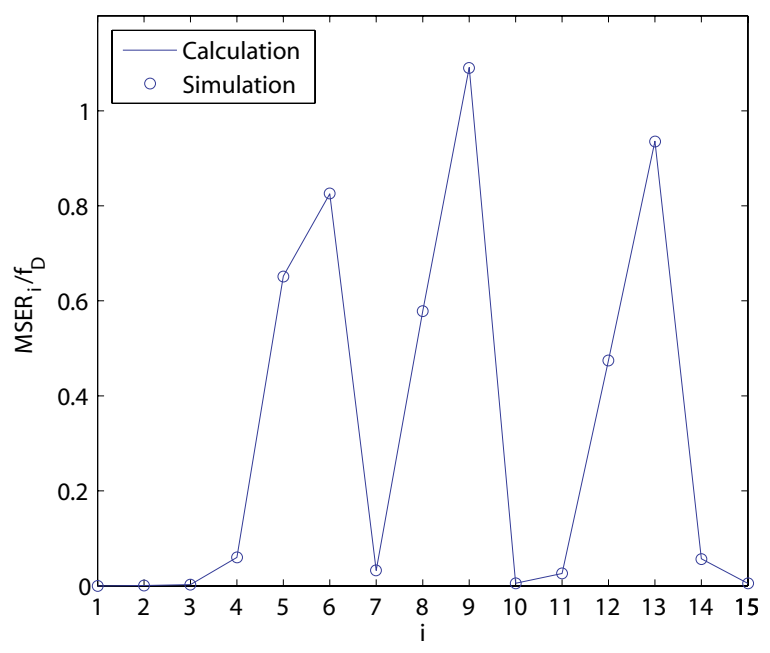

Fig. 2. Simulated vs. calculated modulation state entering rates.

independent processes ${ }^{2}$, which merely gauges how often multiple eigenmodes enter the outage state simultaneously (i.e., have a two-state model with states: all eigenvalues below a threshold and otherwise). Thus, the method in [13] only allows us to determine how often state 1 (outage for all eigenmodes) occurs while all other possible states are not examinable. Fortunately, with the new joint PDF derived in this letter, we can perform a more exact analysis of the joint temporal behavior of the eigenmodes with better accuracy and applicability. In particular, we can evaluate the $\mathrm{MSER}_{i}$, which is defined as the number of times per second that the channel enters a particular state $i$ from any other state. Based on the adaptive system described in this section, it is simple to show that the $\mathrm{MSER}_{i}$ can be written as

$$
\operatorname{MSER}_{i}=\sum_{i \neq j} \frac{P(j)}{\tau} \times P_{j i}=\frac{\sum_{i \neq j} P(i, j)}{\tau} .
$$

A comparison between calculated and simulated $\mathrm{MSER}_{i}$ values is shown in Fig. 2. It is clear that certain states are much more likely to be entered than others.

To determine how long the channel stays in the state $i$, the ASD needs to be evaluated. This is trivial to calculate since

$$
\mathrm{ASD}_{i}=\frac{P(i)}{\mathrm{MSER}_{i}} \text {. }
$$

Our results are shown in Fig. 3. The MSER and ASD are key parameters for setting the feedback/adaptation rate.

\section{B. Impact of Feedback Delay}

Using the FSMC model, we now investigate the impact of mobility on the probability of MSSE for state $i$, denoted $P_{\mathrm{MSSE}, i}$. This is the probability that an inappropriate modulation state is chosen because the channel has changed from state $i$ to another state during the feedback interval $\tau$. This probability can easily be written as:

$$
P_{\mathrm{MSSE}, i}=1-P_{i i} \text {. }
$$

\footnotetext{
${ }^{2}$ Note that such assumption does not reflect reality as the eigenmodes processes are in fact dependent.
}

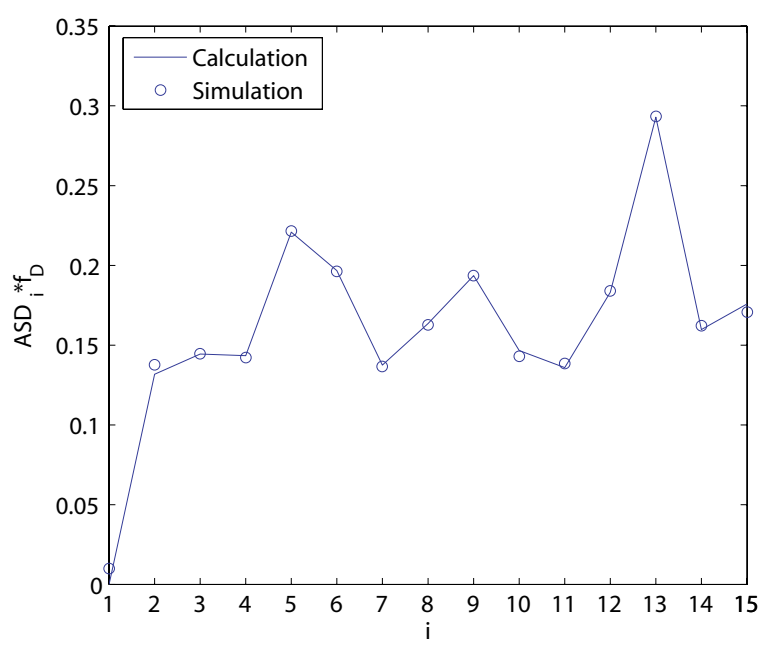

Fig. 3. Simulated vs. calculated average stay duration.

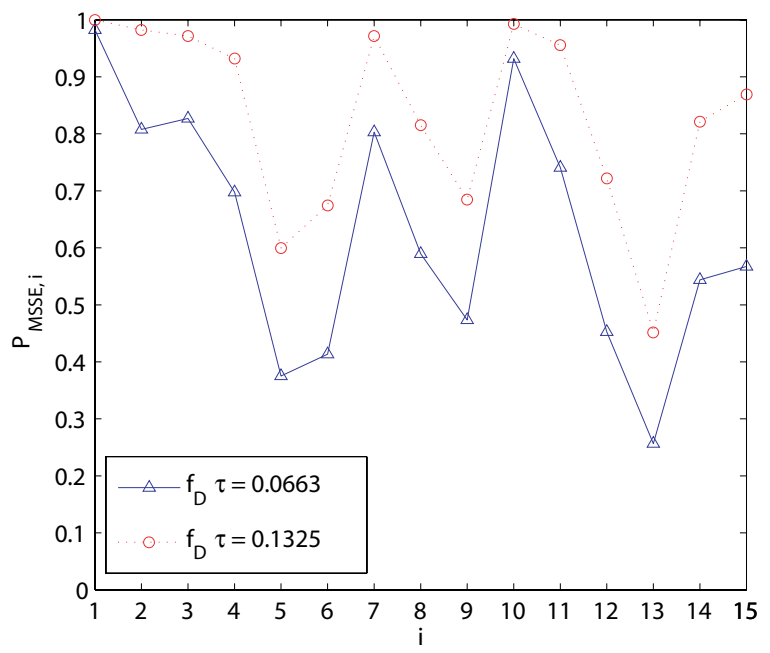

Fig. 4. The probabilities of MSSE for two mobility levels: $f_{D} \tau=0.0663$ and $f_{D} \tau=0.1325$.

We have generated $P_{\mathrm{MSSE}, i}$ for two different mobility levels: $f_{D} \tau=0.0663$ (moderate mobility) and $f_{D} \tau=0.1325$ (high mobility). The results plotted in Fig. 4 show that moderate mobility levels can have a significant impact on modulation selection. The probabilities of choosing an inappropriate modulation state are surprisingly high. The adaptive system is particularly sensitive to time-variation when the eigenmodes occupy certain states (e.g., when $i=1,2,3,4,7$, and 10). We observe that when the system is in state 1 (outage for both eigenmodes), it is almost guaranteed that the channel will switch to another state during the feedback period. Fortunately, from the transition probability calculations, we see that the chance of entering state 1 in the first place is very slim.

\section{CONCLUSION}

In the context of MIMO systems research, we often need to model changes in the channel due to temporal or frequency variation and imperfect estimation. This can often be modeled by a Gaussian perturbation. This paper has two main contributions. Firstly, we have derived the joint PDF for the eigenvalues of the original and the perturbed channel. Secondly, we 
have examined the time-varying characteristics of the MIMO channel. In particular, our joint PDF has been employed to compute the transition probabilities between modulation states in a MIMO-SVD system, and the calculations have been verified through Monte Carlo simulations. From the resultant FSMC, we have analytically computed the MSER and ASD, and observed that system mobility can dramatically affect the adaptive modulation selection performance due to the timecorrelated Rayleigh channel changing rapidly in certain states. Our investigation has provided further insight into the timevarying characteristics of MIMO channels.

\section{ACKNOWLEDGMENT}

The authors would like to thank Dr. Stephan Lawi for helpful discussions regarding his paper [10].

\section{REFERENCES}

[1] B. N. Getu, J. B. Andersen, and J. R. Farserotu, "MIMO systems: optimizing the use of eigenmodes," in Proc. IEEE Int'l. Symp. on Personal, Indoor and Mobile Radio Communications, Sept. 2003, pp. 1129-1133.

[2] M. R. McKay, P. J. Smith, H. A. Suraweera, and I. B. Collings, "On the capacity distribution of OFDM-based spatial multiplexing: exact variance and outage approximation," submitted to IEEE Trans. Inf. Theory.

[3] A. Narula, M. J. Lopez, M. D. Trott, and G. W. Wornell, "Efficient use of side information in multiple-antenna data transmission over fading channels," IEEE J. Sel. Areas Commun., vol. 16, pp. 1423-1436, Oct. 1998.
[4] T. J. Willink, "Improving power allocation to MIMO eigenbeams under imperfect channel estimation," IEEE Commun. Lett., vol. 9, pp. 622624, July 2005.

[5] P. J. Smith and L. M. Garth, "Distribution and characteristic functions of correlated complex Wishart matrices," J. Multivariate Anal., vol. 98, pp. 661-677, Apr. 2007.

[6] X. Zhang, J. Tang, H.-H. Chen, S. Ci, and M. Guizani, "Cross-layer based modeling for quality of service guarantees in mobile wireless networks," IEEE Commun. Mag., vol. 44, pp. 100-106, Jan. 2006.

[7] A. E. Ekpenyong and Y.-F. Huang, "Feedback-detection strategies for adaptive modulation systems," IEEE Trans. Commun., vol. 54, pp. 17351740 , Oct. 2006

[8] I. E. Telatar, "Capacity of multi-antenna Gaussian channels," European Trans. Telecommun. Related Technol., vol. 10, pp. 585-595, Nov. 1999.

[9] W. Konig and N. O'Connell, "Eigenvalues of the Laguerre process as non-colliding squared Bessel processes," Elect. Commun. in Prob. vol. 6, pp. 107-114, 2001.

[10] S. Lawi, "A characterization of Markov processes enjoying the timeinversion property," submitted to Probab. Theory Related Fields, revised Jan. 2006.

[11] S. T. Chung and A. J. Goldsmith, "Degrees of freedom in adaptive modulation: a unified view," IEEE Trans. Commun., vol. 49, pp. 15611571, Sept. 2001.

[12] S. Zhou and G. B. Giannakis, "Adaptive modulation for multiantenna transmissions with channel mean feedback," IEEE Trans. Wireless Commun., vol. 3, pp. 1626-1636, Sept. 2004.

[13] P.-H. Kuo and P. J. Smith, "Temporal behavior of MIMO channel quality metrics," in Proc. IEEE Int'l. Conf. on Wireless Networks, Communications and Mobile Computing, June 2005, pp. 857-862. 